\title{
Nonlinear topological states in the Su-Schrieffer-Heeger model
}

\author{
M. A. Gorlach, A. P. Slobozhanyuk \\ ITMO University, Kronverkskiy, 49, St. Petersburg, 197101, Russia \\ m.gorlach@metalab.ifmo.ru, a.slobozhanyuk@metalab.ifmo.ru
}

PACS 42.50.Ct, 42.70.Qs

DOI $10.17586 / 2220-8054-2017-8-6-695-700$

\begin{abstract}
Topological photonics offers unique functionalities in light manipulation at the nanoscale by means of the so-called topological states which are robust against various forms of disorder. One of the simplest one-dimensional models supporting topological states is the Su-Schrieffer-Heeger model. In this paper, we review the physics of the Su-Schrieffer-Heeger model and its nonlinear counterparts exhibiting self-induced, tunable and many-particle edge states. We discuss the robustness of these states, highlighting their rich potential for nanophotonic and quantum optics applications.
\end{abstract}

Keywords: topological states, nanophotonics, Su-Schrieffer-Heeger model.

Received: 10 November 2017

Revised: 3 December 2017

\section{Introduction}

Research interest in topological photonics that has recently emerged [1-3], is largely driven by the unprecedented possibilities provided by the topological states of light. Such topological states are immune to backscattering on structure imperfections or sharp bends exhibiting a vast range of unusual properties including, for instance, unidirectional spin-locked propagation [1-3].

Very recently, the quest for tunable topological states has given rise to the nonlinear topological photonics, which offers even broader spectrum of opportunities including soliton-like [4-6], self-induced [7,8], tunable [7] and many-particle $[9,10]$ topological states.

Possibly the simplest model of the topological states in one-dimensional systems is provided by the wellcelebrated Su-Schrieffer-Heeger model (SSH) [11,12], which was investigated and realized in various contexts including electronic [11,12], photonic [13-19], plasmonic [20-23], polaritonic [24] and mechanical [25] systems. Though initially the SSH model was applied to explain charge transfer in polymer molecules, it can be also used for describing the physics of artificial photonic and plasmonic structures. In photonic and plasmonic contexts, the SSH model can be implemented with the array of nearest-neighbor coupled cavities with the two alternating tunneling constants as depicted in Fig. 1(a). Plasmonic realization of the SSH model [21-23] allows one to achieve a nanoscale confinement of the edge mode which, however, is subject to the losses inherent to metals. On the contrary, the SSH model based on the all-dielectric platform $[15,16,18]$ is almost loss-free but has worse confinement of light.

Note also that the SSH-type model can be implemented with a zigzag array of identical nanoparticles $[15,18-$ 20,23]. In such geometry, the vector eigenmode problem can be decomposed into two scalar problems, each of which has SSH type. This system can be based on plasmonic [20,23] or all-dielectric [15, 18, 19] platforms that utilize electric dipole [20,23], magnetic dipole [19] or magnetic quadrupole [15,18] resonances of the constituent particles. Topological states in the described model emerge only for the specific range of zigzag angles [15,19].

In this paper, we review the physics of the Su-Schrieffer-Heeger model and its nonlinear generalizations especially focusing on the edge states, their robustness and topological characterization. As we highlight, even such simple model features quite rich physics thus paving a route to the novel nanophotonic applications.

\section{Topological states in the linear SSH model}

The Hamiltonian of the SSH model in the linear case reads:

$$
\hat{H}=\omega_{0} \sum_{m} \hat{n}_{m}-J_{1} \sum_{m}\left[\hat{a}_{2 m}^{\dagger} \hat{a}_{2 m-1}+\hat{a}_{2 m-1}^{\dagger} \hat{a}_{2 m}\right]-J_{2} \sum_{m}\left[\hat{a}_{2 m}^{\dagger} \hat{a}_{2 m+1}+\hat{a}_{2 m+1}^{\dagger} \hat{a}_{2 m}\right]
$$

where $\hat{a}_{m}^{\dagger}$ and $\hat{a}_{m}$ are creation and annihilation operators for the photon in $m^{\text {th }}$ cavity, $\hat{n}_{m}=\hat{a}_{m}^{\dagger} \hat{a}_{m}, J_{1,2}$ are so-called tunneling amplitudes, and $\omega_{0}$ is cavity eigenfrequency. 

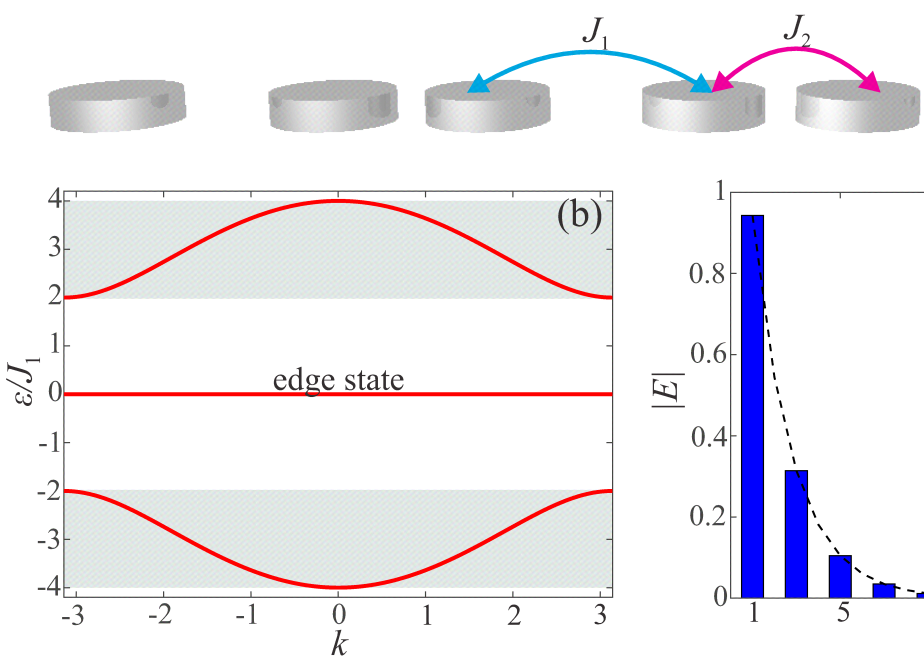

(a)

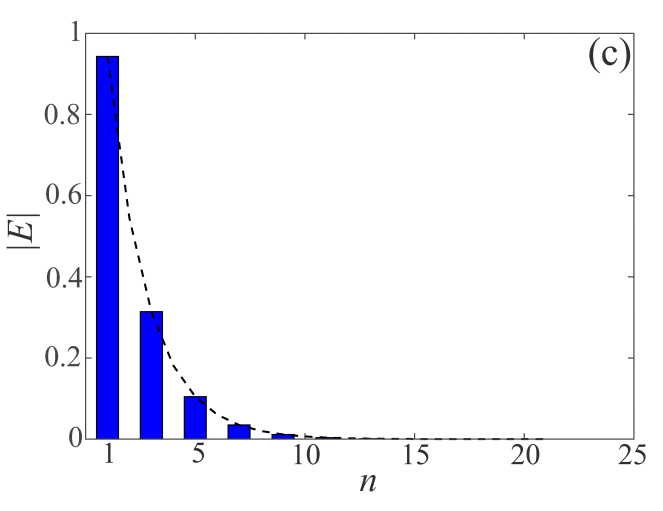

FIG. 1. (a) Array of coupled cavities described by the Su-Schrieffer-Heeger (SSH) model. (b) Energy bands (shaded) and edge states in the SSH model. (c) Field distribution for the the edge state in the $\mathrm{SSH}$ array of $N=21$ coupled resonator. Calculations are performed for $J_{2} / J_{1}=3$

Note that the Hamiltonian commutes with the total number of particles $\hat{N}=\sum_{m} \hat{n}_{m}$. Therefore, the total number of photons in the array is conserved. First, we consider single-photon excitations. The wave function can be searched in the form:

$$
\left|\psi_{1}\right\rangle=\sum_{m} \alpha_{m} \hat{a}_{m}^{\dagger}|0\rangle
$$

where $|0\rangle$ presents the ground state of the system, and coefficients $\alpha_{m}$ are the probability amplitudes to observe a photon in $m^{\text {th }}$ cavity of the array. The eigenvalue equation $\hat{H}|\psi\rangle=\left(\varepsilon+\omega_{0}\right)|\psi\rangle$ yields the system of linear equations for the probability amplitudes $\alpha_{m}$ :

$$
\begin{gathered}
\varepsilon \alpha_{1}=-J_{1} \alpha_{2}, \\
\varepsilon \alpha_{2 m+1}=-J_{2} \alpha_{2 m}-J_{1} \alpha_{2 m+2}, \\
\varepsilon \alpha_{2 m}=-J_{1} \alpha_{2 m-1}-J_{2} \alpha_{2 m+1},
\end{gathered}
$$

where $m=1,2, \ldots$ Quite importantly, exactly the same equations (3)-(5) are obtained in the classical limit when the number of photons is sufficiently large. In the latter case, $\alpha_{m}$ denotes electric field amplitude in $m^{\text {th }}$ cavity. Such correspondence allows one to investigate the physics of the SSH model in fully classical setups, including electronic [11,12], photonic [13-19], plasmonic [20-23], polaritonic [24] and mechanical [25] systems. The bulk energy spectrum of the SSH model is found from Eqs. (4)-(5) using the translational symmetry of the infinite array:

The result reads:

$$
\alpha_{2 m-1}=a e^{i k m}, \quad \alpha_{2 m}=b e^{i k m},-\pi<k<\pi \text {. }
$$

$$
\varepsilon= \pm \sqrt{J_{1}^{2}+J_{2}^{2}+2 J_{1} J_{2} \cos k}
$$

The energy bands of the SSH model with the ratio $J_{2} / J_{1}=3$ are depicted in Fig. 1(b).

However, the SSH model supports not only bulk states Eq. (7), but also the edge state. To illustrate this, we consider a semi-infinite array and apply the full system of equations Eqs. (3)-(5), where Eq. (3) plays the role of the boundary condition. Straightforward calculation yields that the edge state exists under the condition $J_{1}<J_{2}$ (i.e. the edge state emerges at the weak link edge), it has the energy $\varepsilon_{\text {edge }}=0$ and the field distribution $\alpha_{2}=\alpha_{4}=\cdots=\alpha_{2 m}=\cdots=0, \alpha_{2 m-1}=a e^{i k m}$ for $m=1,2, \ldots$ with $e^{i k}=-J_{1} / J_{2}$. The field distribution for the edge state of the SSH model is shown in Fig. 1(c). If the array is terminated by weak links from both edges, two degenerate edge states appear.

Most remarkably, this edge state features some robustness with respect to the various forms of disorder. For instance, the eigenfrequencies of the resonators or tunneling amplitudes in the array can be randomly modulated 

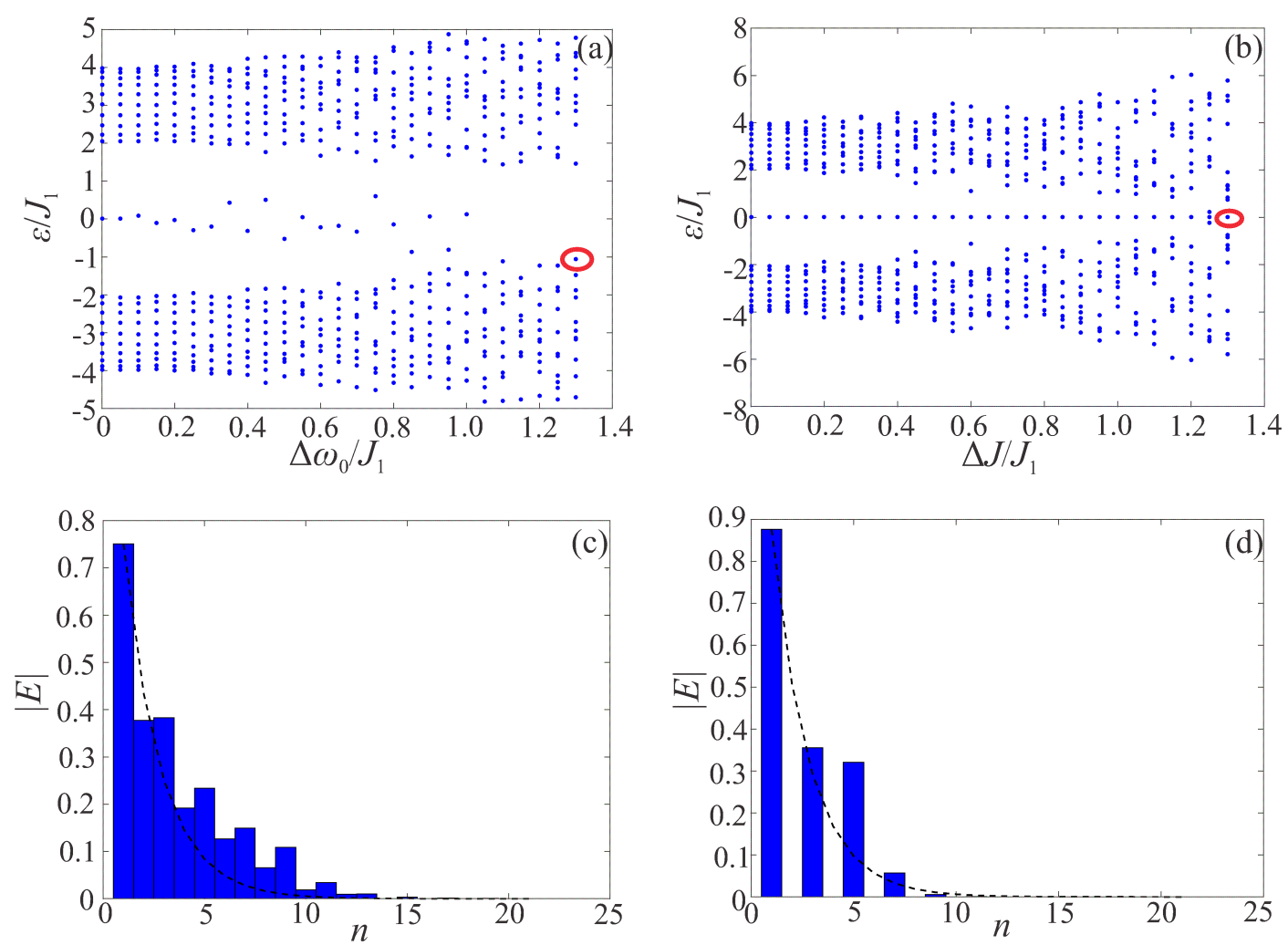

FIG. 2. Energy bands (a,b) and edge states (c,d) in the SSH model with $J_{2} / J_{1}=3$ when the disorder is introduced into the resonator eigenfrequencies $(\mathrm{a}, \mathrm{c})$ or into the tunneling amplitudes (b,d). $\Delta \omega_{0}$ and $\Delta J$ are the standard deviations for the resonator eigenfrequency and tunneling amplitude, respectively. $|E|$ is the field amplitude inside the resonator

with the dispersion $\Delta \omega_{0}^{2}$ and $\Delta J^{2}$, respectively. However, the edge state still persists even though the associated field distribution exhibits some distortions. This important property is further illustrated in Fig. 2.

Futhermore, the very existence of the edge state can be linked to the bulk properties of the system. To elucidate this point, we outline the calculation of the Zak phase [26]:

$$
\gamma=\int_{-\pi}^{\pi} i\left\langle u_{k} \mid \frac{\partial u_{k}}{\partial k}\right\rangle d k,
$$

where $\left|u_{k}\right\rangle$ is the periodic part of the wave function. Since the unit cell contains only two cavities, the periodic part reads $\left|u_{k}\right\rangle=(a, b)^{T}$. With this unit cell choice, $J_{1}$ is an intracell tunneling amplitude, and $J_{2}$ is an intercell tunneling constant. The Zak phase is gauge-independent quantity defined modulo $2 \pi$ that can take two values: $\gamma=0$ for $J_{1}>J_{2}$ and $\gamma=\pi$ for $J_{1}<J_{2}$. Thus, the existence of the edge state is related to the nonzero Zak phase when the edge link is chosen to be the intracell tunneling constant. Note also that the Zak phase depends on the unit cell choice: if $J_{2}$ is considered as an intracell tunneling amplitude, the result for the Zak phase will be the opposite.

\section{Nonlinear SSH models}

The SSH model allows a number of interesting generalizations to the nonlinear domain. Here we will consider two nonlinear systems supporting edge states: classical nonlinear SSH array with the tunneling constants depending on intensity [7,8], and the SSH model in quantum two-particle regime with the effective photon-photon interaction $[9,10]$.

An intuitive way to introduce the tunability in the SSH array of cavities is to make some of the tunneling constants intensity-dependent. The authors of Ref. [7] suggested a specific form of nonlinearity: $J_{2}^{2 m, 2 m+1}=$ $J_{2}+\alpha\left(\left|E_{2 m}\right|^{2}+\left|E_{2 m+1}\right|^{2}\right), J_{1}=$ const, where $E_{m}$ is the field amplitude in $m^{\text {th }}$ resonator. For the zero mode intensity (measured by $A^{2}=\left|E_{1}\right|^{2}+\left|E_{2}\right|^{2}$ ), the system was chosen to be in the trivial regime $J_{1}>J_{2}$ without any 
edge states. However, with an increase of the intensity, the system undergoes a topological transition due to the growth of intercell coupling strength [7]. Such self-induced topological transition is illustrated in Fig. 3(a). Even though the fact of topological transition is suggested by the linear SSH model, the properties of the topological edge state which appears [Fig. 3(b)] strongly differ from its linear counterpart. The reason is that the effective tunneling amplitudes $J_{2}^{2 m,(2 m+1)}$ in the edge state become dependent on the index $m$ due to the mode decay away from the array edge.
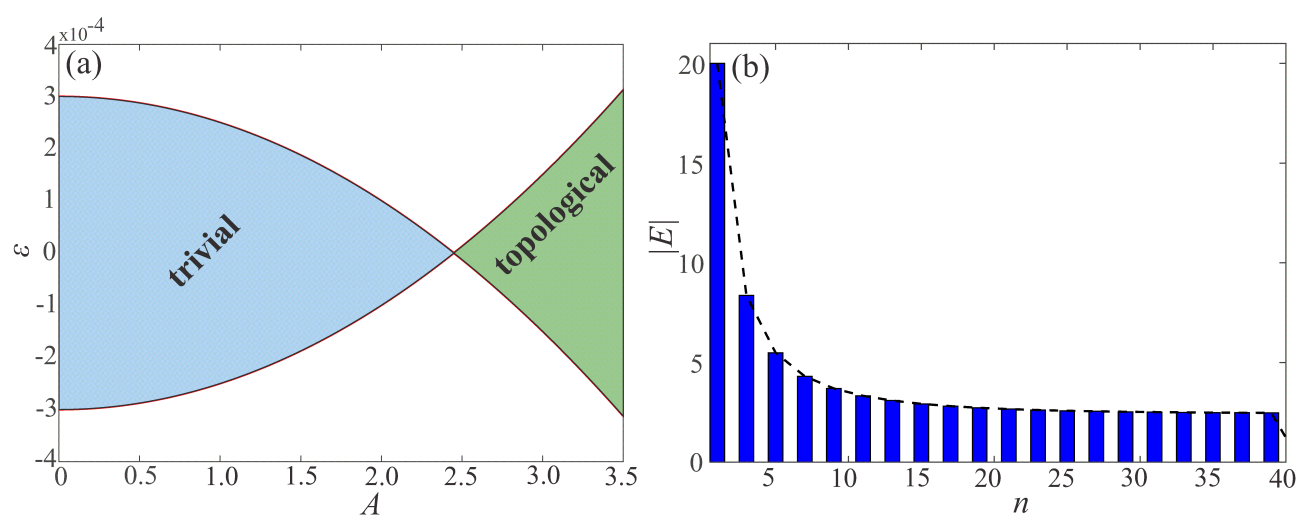

FIG. 3. Self-induced topological transitions and edge states in the nonlinear SSH model with the intercell coupling coefficient depending on intensity. (a) Transition from the trivial regime to the topological one happening due to the intensity increase. (b) Field distribution for the self-induced edge state calculated for $J_{1}=2.3 \cdot 10^{-3}, J_{2}=2.0 \cdot 10^{-3}$ and $\alpha=5.0 \cdot 10^{-5}$

Another interesting system is the two-particle SSH model described by the Bose-Hubbard Hamiltonian $[9,10]$ :

$$
\begin{aligned}
\hat{H}=\omega_{0} \sum_{m} \hat{n}_{m}+U \sum_{m} \hat{n}_{m}\left(\hat{n}_{m}-1\right) & \\
& -J_{1} \sum_{m}\left[\hat{a}_{2 m}^{\dagger} \hat{a}_{2 m-1}+\hat{a}_{2 m-1}^{\dagger} \hat{a}_{2 m}\right]-J_{2} \sum_{m}\left[\hat{a}_{2 m}^{\dagger} \hat{a}_{2 m+1}+\hat{a}_{2 m+1}^{\dagger} \hat{a}_{2 m}\right] .
\end{aligned}
$$

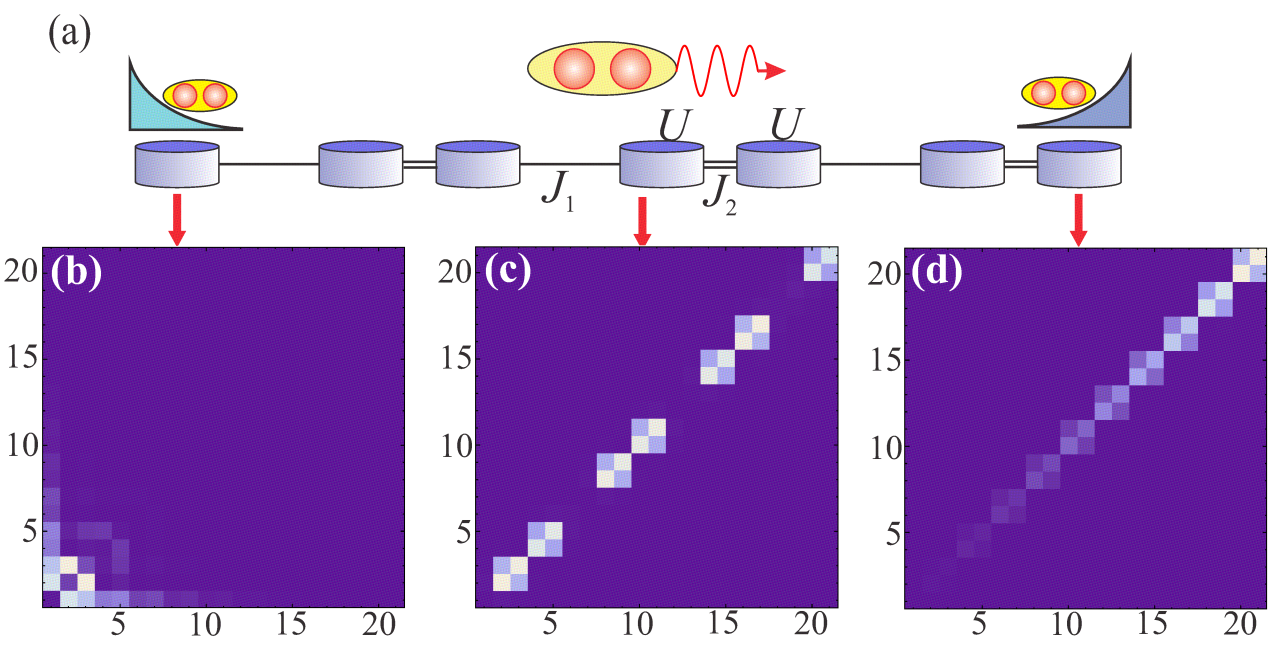

FIG. 4. Two-photon bound bulk and edge states in the SSH array described by Bose-Hubbard model with $J_{2} / J_{1}=5.0, U / J_{1}=8.0$. (a) The schematic of the two-photon bound states. $(b, d)$ Probability distribution for the two-photon edge states localized at the edges of the array with smaller (b) and greater (d) tunneling amplitudes. (c) Probability distribution for the twophoton bulk bound states (doublons). $x$ and $y$ axes show the coordinates of the first and second photon, respectively 
The $U$ term takes into account Kerr-type nonlinearity of the medium inside the resonators. This nonlinearity induces effective photon-photon interactions and gives rise to the photon binding (Fig. 4(a,c)). Quite counterintuitive, bound photon pairs (doublons) emerge even in the case of repulsive nonlinearity $(U>0)[27,28]$ and can appear in the continuum of weakly interacting two-photon states $[29,30]$. In the case of the simple array with $J_{1}=J_{2}$, the doublon edge states are not possible [31,32]. However, in the SSH geometry, the edge states of bound photon pairs can appear not only at the edge with the weak tunneling link (Fig. 4(b)), as in the single-particle case, but also at the edge with the strong tunneling link (Fig. 4(d)) [10] which is a distinctive feature of the two-particle interacting problem. The latter edge states were argued to be topological [10].

\section{Discussion and conclusions}

Despite its conceptual simplicity, the Su-Schrieffer-Heeger model provides qualitatively correct description of the diverse physical phenomena related to electronic, photonic, plasmonic, polaritonic and mechanical systems. In the context of topological states, the most important feature of the SSH model is the emergence of the edge state which has the topological nature being robust against various forms of disorder. This topological state has been observed in a series of experiments with various systems either at the edge of the array or at the domain wall of the two SSH arrays with the different dimerizations.

Nonlinear generalizations of the SSH model feature even more exciting physics. Besides the tunability of the edge states, it becomes possible to realize self-induced topological transitions, soliton-like and many-particle edge states. Quantum topological states can be promising candidates for the variety of quantum optics and quantum information applications including the implementation of qubits and NOON states for quantum precision measurements.

We anticipate that the nonlinear topological SSH-type models which are currently under active investigation can shed new light onto the design of the novel types of nonlinear topological insulators.

\section{Acknowledgements}

This work was supported by the Russian Science Foundation (Grant No. 16-19-10538).

\section{References}

[1] Lu L., Joannopoulos J.D., Soljačić M. Topological photonics. Nature Photonics, 2014, 8 (5), P. 821-829.

[2] Lu L., Joannopoulos J.D., Soljačić M. Topological states in photonic systems. Nature Physics, 2016 , 12 (7), P. 626-629.

[3] Khanikaev A.B., Shvets G. Two-dimensional topological photonics, Nature Photonics, 2017, 11, P. 763-773.

[4] Leykam D., Chong Y.D. Edge solitons in nonlinear-photonic topological insulators. Physical Review Letters, 2016,117 (14), P. 143901.

[5] Chen B.G., Upadhyaya N., Vitelli V. Nonlinear conduction via solitons in a topological mechanical insulator. Proceedings of the National Academy of Sciences of the United States of America, 2014, 111 (36), P. 13004-13009.

[6] Gulevich D.R., Yudin D., et al. Exploring nonlinear topological states of matter with excition-polaritons: Edge solitons in kagome lattice. Scientific Reports, 2017, 7 (1), P. 1780.

[7] Hadad Y., Khanikaev A.B., Alu A. Self-induced topological transitions and edge states supported by the nonlinear staggered potentials. Physical Review B, 2016, 93 (15), P. 155112.

[8] Hadad Y., Vitelli V., Alu A. Solitons and Propagating Domain Walls in Topological Resonator Arrays. ACS Photonics, 2017, 4 (8), P. 1974-1979.

[9] Di Liberto M., Recati A., Carusotto I., Menotti C. Two-body physics in the Su-Schrieffer-Heeger model. Physical Review A, 2016, 94 (6), P. 062704.

[10] Gorlach M.A., Poddubny A.N. Topological edge states of bound photon pairs. Physical Review A, 2017,95 (5), P. 053866.

[11] Su W.P., Schrieffer J.R., Heeger A.A. Solitons in polyacetylene. Physical Review Letters, 1979, 42 (25), P. 1698-1701.

[12] Heeger A.J., Kivelson S., Schrieffer J.R., Su W.P. Solitons in conducting polymers. Reviews of Modern Physics, 1988,60 (3), P. 781-850.

[13] Malkova N., Hromada I., et al. Observation of optical Shockley-like surface states in photonic superlattices. Optics Letters, 2009, 34 (11), P. $1633-1635$.

[14] Schomerus H. Topologically protected midgap states in complex photonic lattices. Optics Letters, 2013,38 (11), P. 1912-1914.

[15] Slobozhanyuk A.P., Poddubny A.N., et al. Subwavelength topological edge states in optically resonant dielectric structures. Physical Review Letters, 2015, 114 (12), P. 123901.

[16] Poli C., Bellec M., et al. Selective enhancement of topologically induced interface states in a dielectric resonator chain. Nature Communications, 2015, 6, P. 7710.

[17] Blanco-Redondo A., Andonegui I., et al. Topological Optical Waveguiding in Silicon and the Transition between Topological and Trivial Defect States. Physical Review Letters, 2016, 116 (16), P. 163901.

[18] Slobozhanyuk A.P., Poddubny A.N., et al. Enhanced photonic spin Hall effect with subwavelength topological edge states. Laser \& Photonic Reviews, 2016, 10 (4), P. 656-664.

[19] Kruk S., Slobozhanyuk A., et al. Edge States and Topological Phase Transitions in Chains of Dielectric Nanoparticles. Small, 2017, 13 (11), P. 1603190.

[20] Poddubny A., Miroshnichenko A., Slobozhanyuk A., Kivshar Y. Topological Majorana States in Zigzag Chains of Plasmonic Nanoparticles. ACS Photonics, 2014, 1 (2), P. 101-105. 
[21] Ling C.W., Xiao M., et al. Topological edge plasmon modes between diatomic chains of plasmonic nanoparticles. Optics Express, 2015, 23 (3), P. 2021-2031.

[22] Cheng Q., Pan Y., Wang Q., Li T., Zhu S. Topologically protected interface mode in plasmonic waveguide arrays. Laser \& Photonics Reviews, 2015, 9 (4), P. 392-398.

[23] Sinev I.S., Mukhin I.S., et al. Mapping plasmonic topological states at the nanoscale. Nanoscale, 2015,7 (28), P. 11904-11908.

[24] St-Jean P., Goblot V., et al. Lasing in topological edge states of a one-dimensional lattice. Nature Photonics, 2017,11 (10), P. $651-656$.

[25] Kane C.L., Lubensky T.C. Topological boundary modes in isostatic lattices. Nature Physics, 2014, 10, P. $39-45$.

[26] Zak J. Berry's Phase for Energy Bands in Solids. Physical Review Letters, 1989, 62 (23), P. $2747-2750$.

[27] Winkler K., Thalhammer G., et al. Repulsively bound atom pairs in an optical lattice. Nature, 2006,441 (7095), P. 853-856.

[28] Valiente M., Petrosyan D. Two-particle states in the Hubbard model. Journal of Physics B, 2008,41 (16), P. 161002.

[29] Zhang J.M., Braak D., Kollar M. Bound states in the continuum realized in the one-dimensional two-particle Hubbard model with an impurity. Physical Review Letters, 2012, 109 (11), P. 116405.

[30] Zhang J.M., Braak D., Kollar M. Bound states in the one-dimensional two-particle Hubbard model with an impurity. Physical Review A, 2013, 87 (12), P. 023613.

[31] Pinto R.A., Nguenang J.P., Flach S. Boundary effects on quantum q-breathers in a Bose-Hubbard chain. Physica D, 2009 , 238 (5), P. 581-588.

[32] Longhi S., Della Valle G. Tamm-Hubbard surface states in the continuum. Journal of Physics: Condensed Matter, 2013, 25 (23), P. 235601 . 